International Journal of Engineering \& Technology, $7(2.24)(2018) 453-456$
International Journal of Engineering \& Technology
SPC
Website: www.sciencepubco.com/index.php/IJET
Research paper

\title{
An Atrial Fibrillation with Normal Limits using Signal Processing- A Review
}

\author{
S.Sathish ${ }^{1}$, K.Mohanasundaram ${ }^{2}$ \\ ${ }^{I}$ Research Scholar (Anna University), Vel Tech Multi Tech, Dr.Rangarajan Dr.Sakunthala Engineering College, Chennai, India. \\ ${ }^{2}$ Professor, Department of Electrical Engineering. Vel Tech Multi Tech, Dr.RANGARAJAN \\ Dr.Sakunthala Engineering College, Chennai, India.
}

\begin{abstract}
Atrial fibrillation is an irregular heartbeat (arrhythmia) that can lead to the stroke, blood clots, heart failure and other heart related complications. This causes the symptoms like rapid and irregular heartbeat, fluttering, shortness of breath etc. In India for every around 4000 people eight of them are suffering from Atrial Fibrillation. P-wave Morphology. Abnormality of P-wave (Atrial ECG components) seen during sinus rhythm are associated with Atrial fibrillation. P-wave duration is the best predictor of preoperative atrial fibrillation. but the small amplitudes of atrial ECG and its gradual increase from isometric line create difficulties in defining the onset of P wave in the Standard Lead Limb system (SLL).Studies shows that prolonged P-wave have duration in patients (PAF) In this Study, a Modified Lead Limb (MLL) which solves the practical difficulties in analyzing the P-ta interval for both in healthy subjects and Atrial Fibrillation patients. P-Ta wave interval and P-wave duration can be estimated with following proposed steps which is applicable for both filtered and unfiltered atrial ECG components which follows as the clinical database trials. For the same the p-wave fibrillated signals that escalates the diagnosis follows by providing minimal energy to recurrent into a normal sinus rhythm.
\end{abstract}

Keywords: Abnormal P-waves, stroke, averaging methods, P-Ta segments, defibrillations circuits.

\section{Introduction}

A Bio signal which bounce the information about the physiological activities of cardio vascular systems is called ECG. These information is very significant clinical aspects about the heart disorders which closely or abnormally deviates from the Normal sinus rhythm[1]. The ECG which is a normal sinus rhythm produced when heart muscle cell contract in retort to the electric impulses caused by natural heart muscle cells SA node. On depolarization, the muscles begin to contract and eject the blood to all parts of the body. The ECG diagnosis reverts most important parameters about the heart which includes about its rhythm, electrical impulses distribution of the throughout the body are intact, Atrium and ventricular chambers expansion, and sometimes ischemic location in the event of a attack myocardial infarction. The ECG signal recorded categorized by five segments which is labelled in electro physiological activities of the heart are P, Q, R, $\mathrm{S}$, and $\mathrm{T}$.

Studies have shown that the frequency range of an ECG signal varies between $0.05 \mathrm{~Hz}$ and $100 \mathrm{~Hz}$ with the dynamic range of amplitude between $1-10 \mathrm{mV}$. The QRS complex is the supreme protuberant waveform which describes about the ventricular depolarization and predominant waveform in the ECG periodic waveforms with normal duration from $0.06 \mathrm{~s}$ to $0.1 \mathrm{~s}$. In India, most of the electrophysiological studies shown only ECG recording system depends on the precise detection of the QRS complex, in particular on ventricular chambers.
ECG analysis depends directly on the Normal sinus rhythm and the data acquired be responsible for all heart related ailments.

The problem associated with the sinus rhythm are ventricular arrhythmias and atrial arrhythmias Most of the studies shown recording and diagnosis system are in ventricular related disorders But there is a minimum focus on atrial arrhythmias. The abnormal in atrial repolarization rises to atrial arrhythmias. In India, atrial fibrillations subjects are prone only eight in numbers out of every 4000 patients are characterized. Atrial fibrillations are termed or defined as because of abnormal p-ta segment[2]. Normal Sinus rhythm waveforms which vary in size, amplitude, and time which is generally obscured because of ventricular activities (depolarization). In Patients suffering from arrhythmias requires defibrillation energy circuits which in lead to resucate patient's life and to project an early preventive treatment.

\subsection{Classification of Atrial Fibrillation}

Atrial fibrillation has different clinical aspects. The categories of atrial fibrillation [3]are classified based on their presence and some significant symptoms are significantly noted in the table 1. The recorded arrhythmias are dynamic over a period of time.

Table 1: Types of Atrial fibrillation

\begin{tabular}{|l|l|l|}
\hline TERMINOLGY & $\begin{array}{l}\text { CLINICAL } \\
\text { FEATURES }\end{array}$ & $\begin{array}{l}\text { ARRYHY-THMIA } \\
\text { PATTERN }\end{array}$ \\
\hline Paroxsyal & $\begin{array}{l}\text { Spontaneous } \\
\text { Terminating }\end{array}$ & Recurrent \\
\hline Persistent & $\begin{array}{l}\text { Not Self } \\
\text { Terminating }\end{array}$ & Recurrent \\
\hline Permenent & $\begin{array}{l}\text { Terminating But } \\
\text { Relapsed }\end{array}$ & Established \\
\hline
\end{tabular}




\subsection{Data collection}

Recording of ECG and data collections has been reaching in advancement for last centuries. The standard 12 Lead ECG system gives an important contribution in collecting the data samples from the different subjects. MIT database[4] which provides and can be extensively used in related with all categories of arrhythmias is noted on the freely available PhysioNet (the MIT Laboratory for Computation Physiology's Web site). It comprises of variety of ECG from different subjects of known heart conditions collectively with normal sinus rhythms and abnormal. These are recorded over a period from 30 minutes to a period using standard limb lead systems and modified limb lead systems. These records are interpreted by clinicians or either, verified by algorithms to aid the further evolution of diagnostic algorithms. Each patient record in the MIT-BIH database, labeled 100 to 124 and 200 to 234, consists of 30 minutes of ECGs sampled at $360 \mathrm{~Hz}[6]$ with 16 bit accuracy.

\section{Preprocessing}

In General, ECG signal[5] acquired is frequently interrupted by different noises and artifacts in due to affect the features of ECG signal. An unwanted problems dues like motion artifacts, muscle noise, electrode artifacts, base line drift line noise, and respiration are intact [6] Hence the preprocessing methods have to be carried out by filtering either analog or digital filters which requires the demand. As part of ECG signal area of interest (P-ta segment) to this work it is in demand to utilize digital filters[7] .These digital filters (Chebyshev filter) which is elliptical nature concentrate in P-ta segment and the same can be normalized.

\subsection{Power Line Interference}

ECG signal when results a poor characteristics may interrupted due to poor grounding and power line interference of $50 \mathrm{~Hz}$ or 60 $\mathrm{Hz}$ AC harmonics,

\subsection{Baseline Wander Noise}

The impact of the baseline wander have the factors like electrode placements and interference between the position of heart and the electrodes. Favor of ECG signal may impulse to change in amplitude and DC baseline drifts which is due to meager conductivity of skin impedance of $1 \Omega$. Figure 1 ECG signal wandering with the base line is removed

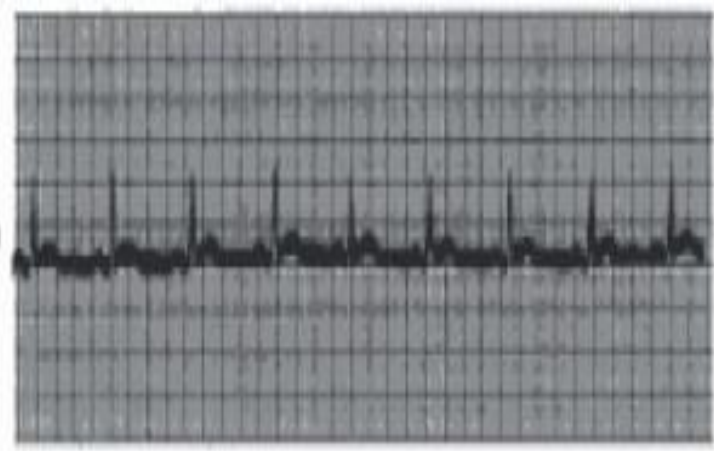

Fig. 1: Removal of Baseline Drift

\subsection{Electromyography artefacts}

EMG artefacts occurs in the ECG signal considered and should be removed by processing methods. When muscles in the surrounding area of the electrodes contract, it would be picked up by the ECG recording systems or acquisition unit. The frequency of this EMG noise is in between $100-500 \mathrm{~Hz}$.

\subsection{Motion Artifacts Noise}

The motion artifacts noise is caused by the improper positioning of the leads. Due to this the movement of the leads produces some error. The fig $\mathbf{2}$ shoes the artifacts signal that can interfere while picking the ECG signals.

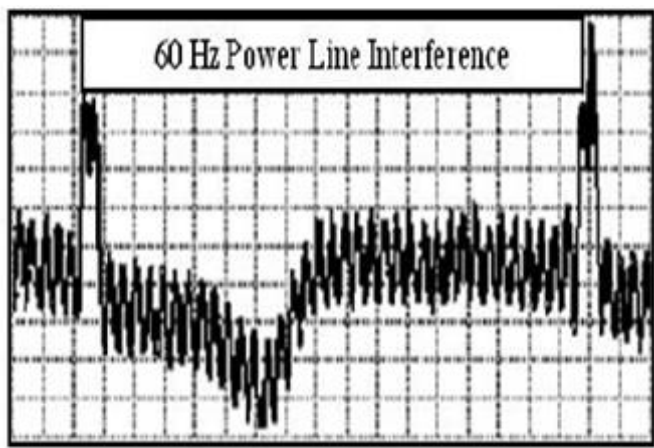

(a)

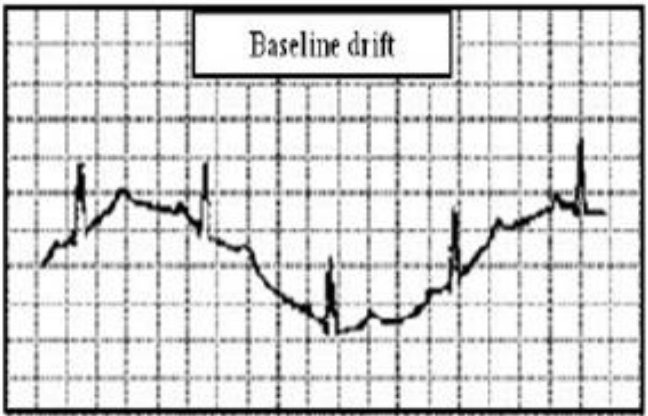

(b)

Fig. 2: Artifact Signals

\section{Methodology}

The ECG samples are diagnosed and the subject arrhythmia's[8] condition are identified and noted for atrial fibrillation. The samples are loaded MATLAB software for analysis in the form of txt (.txt). To diagnose the irregularity signal averaging methods are deployed. A normalization of signal is achieved The stages are as follows Normalisation, Detrending, Smoothening, Wavelet filtering, Windowing[9]

Normalization: The function involved is nyquist frequency which helps to resolve the analog to digital format.

Detrending: To remove the baseline wander noise from the acquired ECG signal.

Smoothening: savitzki-golay filter[10] is recommended to filter acquired ECG data

Wavelet filtering: A part of ECG signal (P-ta ) segment is detached

Windowing: Denoises the signal with the help of empirical mode technique.

\subsection{Signal Averaging}

Signal averaging is a technique used in the time domain, which is done to improvised the acquired ECG. An signal averaging a set of repeated measurements, the signal-to-noise ratio $(\mathrm{S} / \mathrm{N})$ increases ideally in proportion to the square root of the number of measurements. The fig 3 shows the signal averaging of the signal. Noise can be discarded by applying a linear FFT filter. [11]The noise bandwidth intersects the signal bandwidth, and the noise amplitude is greater than the signal. Instance, a FFT Filter will need to discard some of the signal energy in order to remove the noise, thereby distorting the signal. A proper thresholding value should be fixed.. 


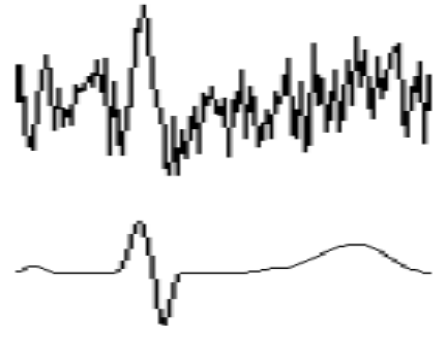

Fig. 3: Signal Averaging of ECG

\subsection{Analysis}

The acquired data were processed by Matlab software. As discussed above signal averaging method with proper threshold values maintained for the acquired normal[12-13] ECG samples. The subjects of average age 20 at the supine position is done. The fig 4 represents the

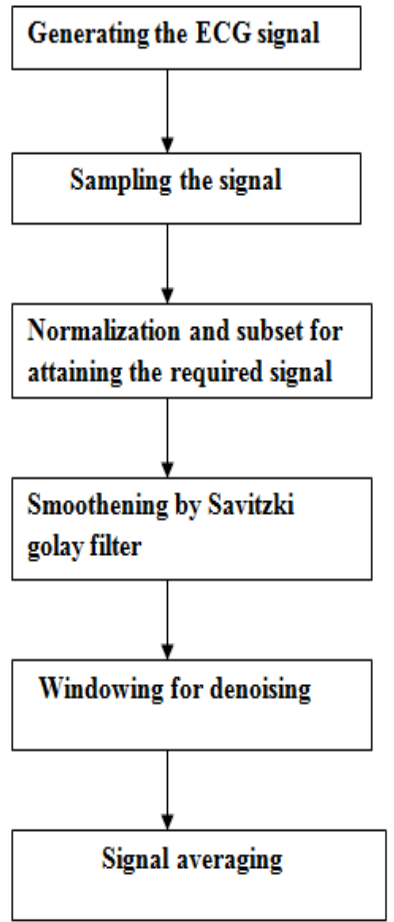

Fig. 4: Processing of ECG signal

Initially 5 male subjects are chosen and the time period and amplitude of normal condition and mimic condition is noted using the Modified limb lead system[14]. Because the normal lead system focus on the ventricular components rather than the ventricular component. The mimic is given by various exercise like push up and running The values are noted in a table below. From the table 2 it is clearly inferred that the time duration of the $\mathrm{P}$-Ta segment decreases in the case of mimic condition than the normal. Usage of MLL enhanced the atrial components which could be seen from the amplitude of $\mathrm{P}$ wave taken for both normal and mimic conditions than 12 lead system.

The table 3 explains about the mean and standard deviation of $\mathrm{P}$ wave and P-Ta interval. [14]The mean age of the subjects chosen was 21 and the mean and standard deviation values are measured

Table 2: Amplitude and Time Period of 5 Male Samples Of Mean Age 20

\begin{tabular}{llllll}
\hline & & Amplitude $(\mathbf{m v})$ & \multicolumn{2}{l}{ Time Period(ms) } \\
\hline Subject & Measurement & Normal & Abnormal & Normal & Abnormal \\
1. & P & 140 & 180 & 72 & 64 \\
& P-Ta & 220 & 300 & 96 & 188 \\
2. & P & 180 & 240 & 96 & 136 \\
& P-Ta & 320 & 440 & 152 & 80 \\
3. & P & 180 & 180 & 72 & 112 \\
& P-Ta & 280 & 300 & 112 & 64 \\
4. & P & 180 & 180 & 80 & 120 \\
& P-Ta & 220 & 320 & 120 & 72 \\
5. & P & 140 & 140 & 96 & 120 \\
\hline
\end{tabular}


And found that the P-Ta amplitude is $124 \mathrm{~ms}$ with a standard deviation of $23 \mathrm{~ms}$ for normal cases and for abnormal cases it ranges from 135 with standard deviation of $30.7 \mathrm{~ms}$.[15]

Table 3: Mean and Standard Deviation of 5 Samples

\begin{tabular}{llllll}
\hline & & Mean & & \multicolumn{2}{c}{$\begin{array}{l}\text { Standard } \\
\text { deviation }\end{array}$} \\
\hline & Measurem & Norm & Abnor & Norm & Abnor \\
& ent & al & mal & al & mal \\
AMPLITU & P & 164 & 184 & 21.9 & 35.7 \\
DE (mv) & P-Ta & 254 & 329 & 63.5 & 77.0 \\
& & & & & \\
TIME & P & 83 & 74 & 12.1 & 8 \\
PERIOD & P-Ta & 124 & 135 & 23.0 & 30.7 \\
(ms) & & & & & \\
\hline
\end{tabular}

The fig 5 shows is the input signal that is taken from the subject using modified limb lead sytem which shows a enhanced P-Ta interval when compared to conventional 12 lead system.

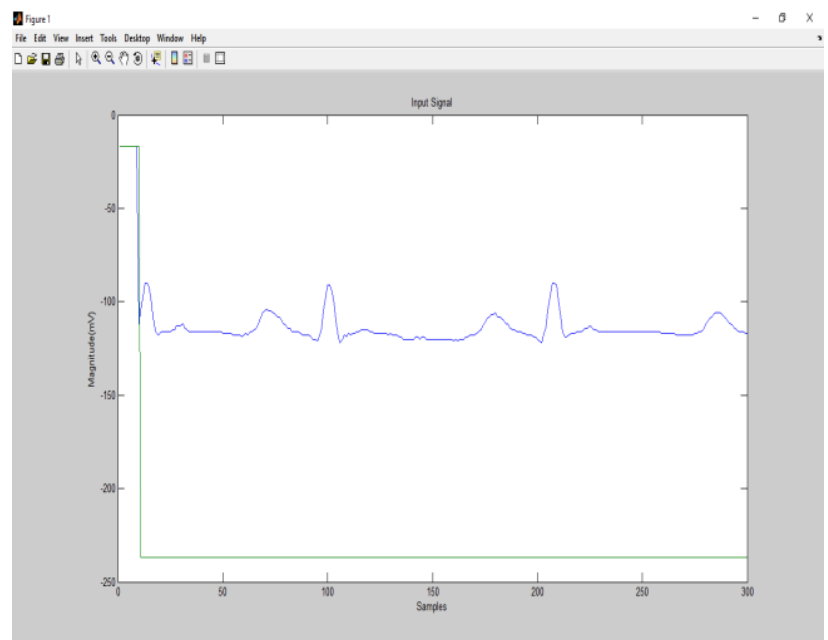

Fig. 5: ECG Wave Obtained Using Modified Limb Lead System

The fig 6 shows the first step of the processing a signal is preprocessing which includes filtering and the normalization. The filtering is done to remove the noises like EMG interference, power line interference, base line wandering and baseline drift. The normalization of the signal is done to bring the values of the signal to the manageable limits.

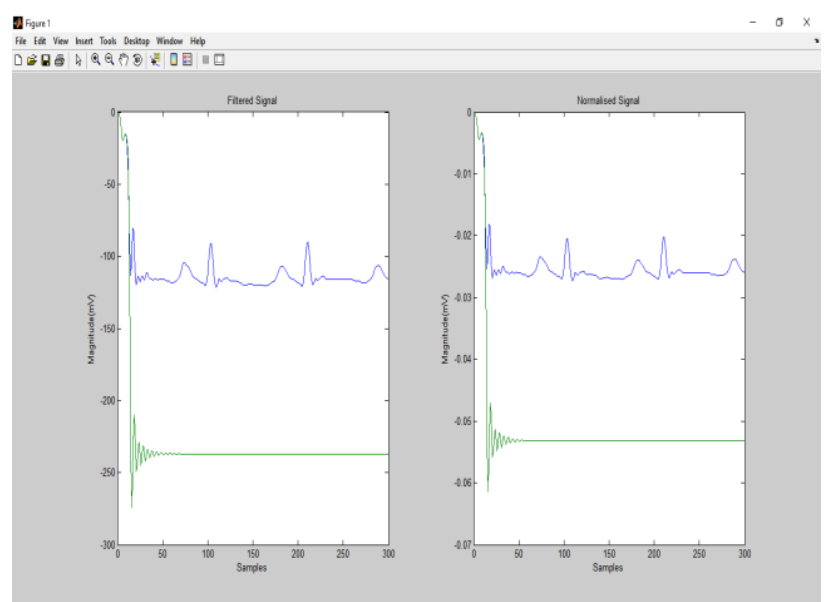

Fig. 6: The Filtered and Normalized ECG Signal

\section{Result}

The atrial Ta wave is clearly enhanced using Modified limb lead system. The windowing and signal averaging technique shows segmented P-Ta interval. For the normal subjects the time interval
And the amplitude of the $\mathrm{p}$ wave increases, which was about 164 $\mu \mathrm{v}$ with standard deviation of $\pm 21.9 \mu \mathrm{v}$ for normal condition and $184 \mu \mathrm{v}$ with a standard deviation of $25.7 \mu \mathrm{v}$.

is $124 \pm 23 \mathrm{~ms}$ and of the fibrillated ECG is $134 \pm 30 \mathrm{~ms}$. On comparing the time intervals of normal and atrial fibrillation patients there is significant change in time duration. It is clearly seen that the time interval of P-Ta interval abnormally varies with atrial fibrillation patients when compared with normal subjects. A database created using this can be used for the clinical diagnosis in the hospitals for the diagnosis of atrial fibrillation.

\section{References}

[1] Einthoven W. "The different forms of the human electrocardiogram and their signification." Lancet 1912; 1: 85361

[2] Atrial fibrillation in a tertiary care institute A prospective study2012 Sep; 64(5): 476-478.

[3] K. Konings, C. Kirchhof, J. Smeets, H. Wellens, et al., "Highdensity mapping of electrically induced atrial fibrillation in humans, Circulation, vol. 89, pp. 1665-80, 1994.

[4] ECG Acquisition, Storage, Transmission, and Representation Gari D. Clifford and Matt B. Oefinger.

[5] G. B. Moody, R. G. Mark, and A. L. Goldberger, "PhysioNet: A w web based resource for the study of physiological signals," IEEE Engineering in Medicine and Biology Mag., vol. 20, no. 3, pp. $70-75,2001$.

[6] Abboud, S., and O. Barnea, "Errors Due to Sampling Frequency of Electrocardiogram in Spectral Analysis of Heart Rate Signals with Low Variability," Computers in Cardiology, Vol. 22, September 1995, pp. 461-463.

[7] Enhancement of ECG Signal by using Digital FIR Filter B. Jagadiswara Rao1, A. Usharani2., International Journal of Science and Research (IJSR)., India.

[8] Sivaraman J, Uma G, Venkatesan S, Umapathy M, Dhandapani VE. "A novel approach to determine atrial repolarization in electrocardiograms." J Electrocardiol 2013; 46: e1

[9] Oppenheim, A. V., and R.W. Schafer, Discrete-Time Signa Processing, Englewood Cliffs, NJ: Prentice-Hall, 1999.

[10] Clifford, G. D., and P. E. McSharry, "Method to Filter ECGs and Evaluate Clinical Parameter Distortion Using Realistic ECG Model Parameter Fitting," Computers in Cardiology, Vol. 32, 2005

[11] C. Li, C. Zheng, and C. Tai, "Detection of ECG characteristic points using wavelet transforms," IEEE Trans. Biomed. Eng., vol. 42, no. 1, Jan.1995

[12] Sivaraman J, Uma G, Venkatesan S, Umapathy M, Keshav Kumar N. "A study on atrial Ta wave morphology in healthy subjects: Anapproach using P wave signal-averaging method." Med Imaging Health Inf 2014; 4: 675-80

[13] Joseph Ackora-Prah, Anthony Y. Aidoo "An Artificial ECG Signal Generate Function in MATLAB", Applied Mathematica Sciences, Vol.7, 2013, no. 54, 2675 - 2686 HIKARI Ltd

[14] Sivaraman J, Uma G, Venkatesan S, Umapathy M,Dhandapani VE. "Normal limits of ECG measurements related to atria activity using a modified limb lead system." Anatol J Cardiol 2014 Feb26. Epub ahead of print.

[15] Fujiki, H. Nagasawa, M. Sakabe, K. Sakurai, et al., "Spectra characteristics of human atrial fibrillation waves of the right atrial free wall with respect to the duration of atrial fibrillation and effect of class I antiarrhythmic drugs," Jpn Circ J, vol. 65, pp. 1047-51, 2001.

[16] S.V.Manikanthan and T.Padmapriya "Recent Trends In M2m Communications In $4 \mathrm{~g}$ Networks And Evolution Towards 5g", International Journal of Pure and Applied Mathematics, ISSN NO:1314-3395, Vol-115, Issue -8, Sep 2017

[17] T.Padmapriya, S.V.Manikanthan, "An enhanced distributed evolved node-b architecture in 5G tele-communications network' , International Journal of Engineering \& Technology, DOI: 10.14419/ijet.v7i2.8.10419, ISSN NO:2227-524X, Vol-7, No.2.9(2018) 